\title{
Integrating technical and political views for a sustainable European Distributed Infrastructure on Population Health
}

Alicia Padron-Monedero ${ }^{* *}$ (D), Rodrigo Sarmiento Suárez ${ }^{1}$, Petronille Bogaert ${ }^{2}$, Linda Abboud ${ }^{2}$, Herman Van Oyen ${ }^{2,3}$, Hanna Tolonen ${ }^{4}$, Mariken J. Tijhuis ${ }^{5}$, Luigi Palmieri', Romana Haneef ${ }^{7}$, Anne Gallay ${ }^{7}$, Luis Lapao ${ }^{8}$, Paulo Jorge Nogueira ${ }^{9}$, Thomas Ziese ${ }^{10}$, Stefanie Seeling ${ }^{10}$, Jakov Vukovic ${ }^{11}$ and Isabel Noguer-Zambrano ${ }^{1}$

\begin{abstract}
Background: Non-Communicable diseases (NCD) are the main contributors to mortality and burden of disease. There is no infrastructure in Europe that could provide health information (HI) on Public Health monitoring and Health Systems Performance (HSP) for research and evidence-informed decision-making. Moreover, there was no EU and European Economic Area Member States (EU/EEA MSs) general consensus, on developing this initiative and guarantee its sustainability. The aim of this study is to analyze the integration of technical and political views made by the Joint Action on Health Information (InfAct; Information for Action) and the results obtained from those activities, in terms of advice and national and institutional support to develop an integrated and sustainable European Distributed Infrastructure on Population Health (DIPoH) for research and evidence-informed policy-making.

Methods: InfAct established two main boards, the Technical Dialogues (TDs) and the Assembly of Members (AoM), to provide a platform for discussion with EU/EEA MSs to establish a sustainable infrastructure for HI: 1) The TDs were composed by national technical experts (NTE) with the aim to discuss and provide feedback about scientific aspects, feasibility and EU-added value of the infrastructure proposed by InfAct. 2) The AoM gathered country representatives from Ministries of Health and Research at the highest political level, with the aim of providing policy-oriented advice for the future political acceptance, support, implementation, and development of InfAct's outcomes including DIPoH. The documentation provided for the meetings consisted in Fact-Sheets, where the main results, new methods and proposals were clearly exposed for discussion and assessment; altogether with more extended information of the DIPoH. The documentation was provided to national representatives within one more before each TD and AoM meeting. The Agenda and methodological approaches for each TD and AoM meeting consisted in the presentations of the InfAct outcomes extending the information provided in the Fact-Sheets; followed by a non-structured interaction, exchange of information, discussion and suggestions by the MSs representatives.

The outcomes of the non-structured discussions were collected in Minutes of the TD and AoM meetings, and the final version was obtained with the consensus of all participants. Additionally, structured letters of political support were provided to the AoM representatives, for them to consider providing their MS written support for DIPoH.
\end{abstract}

\footnotetext{
* Correspondence: a.padronm@isciii.es

${ }^{1}$ National School of Public Health, Instituto de Salud Carlos III. Av/ Monforte de Lemos 5, 28029 Madrid, Spain

Full list of author information is available at the end of the article
}

(c) The Author(s). 2022 Open Access This article is licensed under a Creative Commons Attribution 4.0 International License, which permits use, sharing, adaptation, distribution and reproduction in any medium or format, as long as you give appropriate credit to the original author(s) and the source, provide a link to the Creative Commons licence, and indicate if changes were made. The images or other third party material in this article are included in the article's Creative Commons licence, unless indicated otherwise in a credit line to the material. If material is not included in the article's Creative Commons licence and your intended use is not permitted by statutory regulation or exceeds the permitted use, you will need to obtain permission directly from the copyright holder. To view a copy of this licence, visit http://creativecommons.org/licenses/by/4.0/ The Creative Commons Public Domain Dedication waiver (http://creativecommons.org/publicdomain/zero/1.0/) applies to the data made available in this article, unless otherwise stated in a credit line to the data. 
Results: NTE, within the TDs, considered that DIPoH was useful for technical mutual learning and cooperation among and within countries; although they considered that the technical feasibility to uptake InfAct deliverables at the national and EU level was complex. The AoM focused on political support, resources, and expected MSs returns. The AoM representatives agreed in the interest of setting up an integrated and sustainable $\mathrm{H}$ infrastructure and they considered $\mathrm{DIPOH}$ to be well-articulated and defined; although, some of them, expressed some barriers for providing DIPoH political support. The AoM representatives stated that the AoM is the most suitable way to inform EU MSs/ACs about future advances of DIPoH. Both boards provided valuable feedback to develop this infrastructure. Eleven countries and sixteen institutions supported the proposal, either by letters of political support or by signing the Memorandum of Understandings (MoU) and three countries, additionally, provided expression of financial commitment, for DIPoH to be added to the ESFRI 2021 roadmap.

Conclusions: TDs and AoM were key forums to develop, advise, advocate and provide support for a sustainable European research infrastructure for Population Health.

Keywords: InfAct, Health information, Health systems performance, Non- communicable diseases, Distributed infrastructure on population health

\section{Background}

The Council of the European Union (EU) [1] recommended in 2013 to the Commission of the EU and to the Member States (MSs) to establish an integrated and sustainable EU Health information system (HIS) focussed on Population Health and Health Systems Performance (HSP). However, this recommendation was not included in the agenda of main priorities of the MSs. Moreover, there was no general consensus at the EU and European Economic Area Member States (EU/EEA MSs) on how to develop this initiative and guarantee its sustainability. Although, some level of agreement had been previously outlined [2-4] (the health information system infrastructure should facilitate interaction between networks and experts, it should be distributed although the coordination could be performed by a central hub, and the infrastructure should provide access to high quality interoperable data for population health research and evidence based policies) [2-4].

A previous initiative to establish a shortlist of public health indicators serving as the core of a European public health monitoring system started in 1998 in response to a European Commission (EC) 's call. Several EUfunded projects developed the list of European Core Health Indicators (ECHI), and the first version of the ECHI shortlist was approved by the EC and the EU MSs in 2005. In 2008, a project funded by the Second Health Programme of the EC (2008-2013) stated that the EC and the EU MSs could put the indicators into practice with the aim of monitoring and comparing a limited number of health outcomes among EU countries, and to support evidence-informed policy-making [5]. However, this valuable initiative lacked sustainability at the EU level, due to an absence of updating and a definite governance structure beyond its funding period.
Another project, funded by the Third Health Programme of the EC (2014-2020), "Bridging Information and Data Generation for Evidence-based Health policy and research" (BRIDGE Health), was carried out from 2015 to 2017 with the aim to assess different structural and institutional options to develop a sustainable and integrated EU-HIS for both public health and research purposes. The BRIDGE Health project successfully developed the first proposal for the core elements (scope, tasks, activities, and governance) for an EU-HI research infrastructure [2-4].

Building on the Council of the EU recommendation [1] and the experience and results from the aforementioned initiatives $[2,5]$, the Joint Action on Health Information (InfAct, Information for Action) [6] was launched in March 2018 to run for three years (20182021). InfAct brings together 40 institutions from 28 EU/EEA MSs with the aim of developing an integrated and sustainable EU-HI infrastructure supporting country knowledge and capacities for health research and evidence-informed policy-making. To involve political and Technical Experts from EU/EEA MSs was considered necessary, in order to decide collaboratively whether an integrated and sustainable European Distributed Infrastructure on Population Health (DIPoH) was the best option for a EU-HI infrastructure among the several options investigated [2], and to gather their political and technical advice, acceptance and longterm support.

This paper aims to analyze the activities within InfAct to integrate technical and political views and the results obtained from those activities, in terms of advice and support, from EU/EEA MSs for innovate HI and setting up DIPoH, as a way forward to ensure sustainability beyond EU funded projects. 


\section{Methods}

InfAct has established two main boards: Technical Dialogues (TDs) and the Assembly of Members (AoM). The main objectives of these boards were to ensure engagement with EU/EEA MSs, to discuss, advice and generate consensus and support for a future sustainable EU-HI infrastructure. Both boards were established as follows:

\section{The Technical Dialogues (TDs)}

TDs were composed by National Technical Experts (NTE), as representatives from EU/EEA MSs, and nominated from Ministry of Health $(\mathrm{MoH})$ and Ministry of Research (MoR) authorities. InfAct's recommendations for selecting the NTE were flexible to adapt to each MS singularity. InfAct's recommendations included, as a general concept, to be in charge of their national HIS or working directly with them. A total of 15 EU/EEA MSs NTE representatives participated actively in the meetings. The participating countries were: Austria, Belgium, Croatia, Estonia, Finland, France, Germany, Italy, Ireland, Malta, Netherlands, Norway, Portugal, Spain, and Serbia.

The specific objectives of the TDs were: to provide technical feedback on activities of the InfAct project team, to assess the added value and feasibility to make DIPoH operational, and to raise awareness among decision makers.

Two TD meetings were held: October 2019 (Madrid) and September 2020 (virtual conference due to COVID19 travel restrictions).

The InfAct outcomes are the products of each Work Package of the InfAct project team, in relation to DIPoH four core activities: "1. provide a one-stop-shop for population health data, 2. develop innovative methods, 3. build capacity, and 4. develop knowledge translation research" [7]. Each InfAct outcome was described and summarized, within a structured format, by the InfAct's team responsible, in the form of Fact Sheets. In those Fact-Sheets, the main results, including proposals, guidelines, tools, and methods were clearly exposed for discussion and assessment (Table 1) [8, 9]. The Fact-Sheets were provided to the NTE within one more before each TD. Additionally, more extended information of the proposed new HI research infrastructure called DIPoH was also distributed [7]. DIPoH is designed as a $\mathrm{HI}$ distributed infrastructure to provide high quality EU information on population health, health determinants, and healthcare systems for innovative research and evidenceinformed policy-making [7].

The agenda and methodological approach for the meetings consisted of presentations by InfAct beneficiaries of the technical outcomes of InfAct extending the information provided in the Fact-Sheets [8, 9]; followed by a non-structured interaction, exchange of information, discussion and suggestions by the NTE.

The outcomes of the non-structured discussions were summarized, integrated and collected in Minutes of the TD. The NTE received a draft of the minutes one month after each meeting to provide additional comments and suggestions and to accept the final version by consensus.

\section{The Assembly of Members (AoM)}

The AoM provided a platform between InfAct and national government representatives (one from the $\mathrm{MoH}$

Table 1 Joint Action on Health Information Fact Sheets. Technical Dialogues meetings, 2019 and 2020 [8, 9]

\begin{tabular}{|c|c|}
\hline Fact-Sheet & Topic \\
\hline 1 & Prioritizing health information at national level \\
\hline 2 & Capacity building activities under the European Health Examination Survey \\
\hline 3 & Contributions to European Health Information Training Program (EHITP) \\
\hline 4 & Health Information Training Course and roadmap for sustainability \\
\hline 5 & Connecting health information system's stakeholders through national nodes \\
\hline 6 & Health data collection methods and procedures \\
\hline 7 & Guidance for health reports \\
\hline 8 & A sustainable ECHI shortlist \\
\hline 9 & Innovative use of data sources \\
\hline 10 & Use of artificial intelligence for public health surveillance \\
\hline 11 & Burden of disease \\
\hline 12 & Methodological guidelines to estimate health indicators using linked data and machine learning techniques \\
\hline 13 & Non-health related EU databases for health surveillance. Case study industrial pollution and cancer \\
\hline 14 & Composite health indicators for monitoring NCD: Hospital admissions and mortality ratio \\
\hline 15 & Interoperability \\
\hline
\end{tabular}


and one from the MoR) to discuss the strategic vision for DIPoH and InfAct outcomes [7, 9] and to provide feedback, political guidance, and support to their future short and long term implementation. Additionally, the AoM has set the basis for a permanent governance body of DIPoH [9].

A total of 30 representatives of $\mathrm{MoH}$ and $\mathrm{MoR}$ from 22 EU/EEA MSs have participated actively in the meetings. The participating countries included: Austria, Belgium, Bosnia and Herzegovina, Czech Republic, Croatia, Estonia, Finland, France, Greece, Iceland, Ireland, Italy, Lithuania, Malta, Norway, Netherlands, Portugal, Spain, UK, Romania, Serbia, and Luxembourg.

Originally three meetings were scheduled to be held during the project, the first one to present InfAct to the representatives and to accept the Terms of Reference of the AoM and the other two meetings after the two TDs. However, it was necessary the organization of two additional meetings with the aim of discussing: the impact of COVID-19 pandemics in the project, the proposal for DIPoH [7], the European Strategy Forum on Research Infrastructures (ESFRI) application for the $\mathrm{DIPoH}$ and the setting up of PHIRI "as a practical rollout of DIPoH in the pandemic context" $[9,10]$. Thus, a total of five AoM meetings were held. The first two meetings were face-to-face in March 2019 in Madrid and November 2019 in Brussels. The last three meetings (June 2020, October 2020, and May 2021) were held virtually due to the COVID-19 pandemic's travel restrictions.

The Fact-Sheets $[8,9]$, the Minutes of the TD meetings, and more extended information of the DIPoH [7] were provided to the AoM representatives one month before each AoM meeting (Table 1).

During the AoM meetings, non-structured discussions were held based on: InfAct main outcomes presented by InfAct beneficiaries, Fact-Sheets and TDs Minutes with technical recommendations and suggestions (Fig. 1). All participating MSs representatives were able to provide their inputs related to the new proposed research infrastructure (DIPoH).

The outcomes of the non-structured discussions from the AoM representatives about the strategic vision for DIPoH including the feedback, advice, and requirements for their support were summarized, integrated and detailed in the Minutes of each AoM. Within one month of each meeting the AoM representatives had the chance of reviewing the minutes and to provide additional comments and suggestions. All participants accepted the final versions [9].

Additionally, structured letters of political support were provided to the AoM representatives, for them to

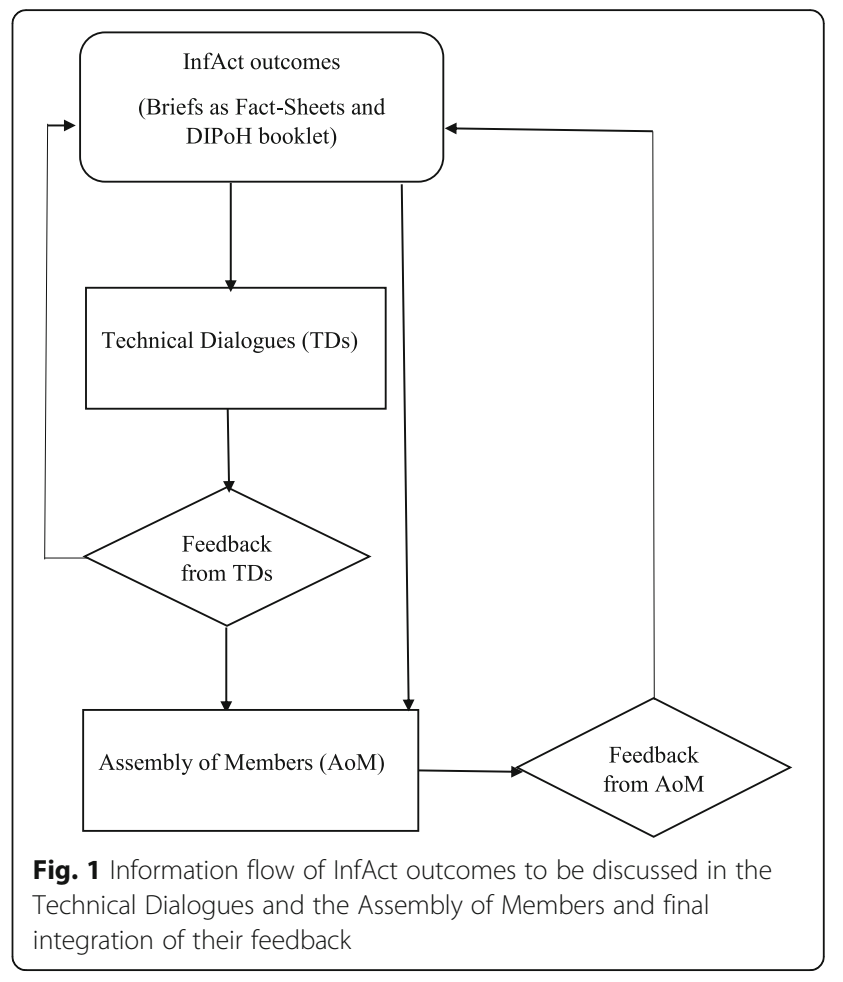

consider providing their MS support for DIPoH to be added to the ESFRI 2021 roadmap.

\section{Results}

\section{The Technical Dialogues}

NTE participating in TDs were able to share clear and realistic needs, gaps in $\mathrm{HI}$ (in their own country and at EU level), and a wide range of views and concerns for the future. The NTE's comments and suggestions were always submitted to the research and health authorities' for them to make the final decision about whether to provide political support to InfAct's outcomes including DIPoH; however, it is important to highlight that the NTE technical assessments were positive regarding the added value of InfAct's outcomes.

Regarding InfAct's main outcomes, the TD meetings reached the following consensus, that are detailed in the agreed minutes [9]:

- InfAct outcomes provided highly valuable tools and guidelines to be used nationally by experts.

- The proposed research infrastructure DIPoH is useful for technical mutual learning and cooperation among and within countries.

- NTE considered obtaining quality, accurate and robust health data as important and desirable goals.

- The feasibility to take up InfAct's deliverables into HIS at national and EU level was considered 
complex. The main difficulties raised were: the different data quality and HI methods between countries, different functional and organizational approaches stablished in different countries, and different MSs' interests.

- The capacity building activities provided by InfAct were considered valuable and needed at EU level.

- Additionally, the European General Data Protection Regulation (GDPR) was considered a possible technical concern in terms of $\mathrm{HI}$ interoperability between the countries in general. However, it was acknowledged that InfAct had made an assessment of GDPR interpretation and implementation in different countries and delivers possible options for the interoperability of health data in the EU respecting GDPR [11]. On the other hand, the NTE considered the anonymization of data an important concern. They suggested the elaboration of consensus guidelines about anonymization to be considered in DIPoH's data management plan.

Overall, the technical outcomes presented by InfAct in the TD meetings were considered valuable for establishing priorities to advise decision makers.

The DIPoH proposal and its submission to the ESFRI roadmap were welcomed, but there were discussions about difficulties that may be faced. Country-specific organizations, financial issues and political positions were highlighted as challenging factors to implement $\mathrm{DIPoH}$.

\section{The Assembly of Members}

The AoM meetings reached the following consensus that are detailed in the agreed minutes [9]:

- All countries' representatives agreed in the interest of setting up an integrated and sustainable HI infrastructure gathering population health information in order to support health research and evidence-informed policy-making.

- DIPoH was welcomed, and it was considered to be well-articulated and defined. Specifically, it was appreciated that several options for an EU-HIS had been investigated, providing their advantages and disadvantages [2]. It was also valued that the ESFRI roadmap application for DIPoH included different financing options as requested by the MSs. In summary, eleven countries and sixteen institutions supported the proposal, either by letters of political support or by signing the Memorandum of Understanding (MoU) and three countries, additionally, provided expression of financial commitment, for DIPoH to be added to the ESFRI 2021 roadmap.
- EU institutional interaction was highlighted as a key necessary value.

- The representatives valued the setting up of the Population Health Information Research Infrastructure (PHIRI) (funded by the DirectorateGeneral for Research and Innovation Grant Number 101018317) [10] as a practical roll out of DIPoH for COVID-19 that will further implement DIPoH infrastructure and services.

- The representatives considered that the AoM, as a forum, was the most suitable way to inform all EU/ EEA MSs on DIPoH's progress and InfAct's outcomes.

The main barriers for providing DIPoH political support were: future financial support, the commitment of the different budget providers among government departments (Research or Health), different strategic interests depending on departments, MSs internal and bureaucratic procedures, and the need for a clear benefit at national level. Finally, some MSs still had some concerns if a Research Infrastructure is the ideal format for an EU-HIS. They suggested that they would instead prefer an enlargement of the scope of ECDC to cover areas beyond infectious diseases, including chronic diseases and HSP. The need for wide country participation to maximize the added value of $\mathrm{DIPoH}$ was also stressed to increase country adherence to the initiative.

\section{Discussion}

As a result of the discussions and the feedback provided by the participants from the TDs and the AoM boards, some important conclusions were drawn:

First, the early involvement of both the technical and political boards (in a multi-sectorial approach since the beginning of the project) had important benefits in guiding the development of DIPoH to support evidenceinformed policy-making, because the boards provided the necessary feedback and suggestions about the requirements they would find appropriate for DIPoH's future support, acceptance, and sustainability. This conclusion can be drawn from the members' statement (expressed during the meetings and collected in the minutes) that their recommendations were needed to improve the proposal, in order to accept and support the long-term establishment of $\mathrm{DIPoH}$ in some countries. The proposal's progressive evolution, including the members' recommendations from each meeting, was appreciated and acknowledged by the representatives when the final proposal was presented in the last AoM meeting.

Second, to involve the NTEs and the political authorities in a multi-sectorial approach was beneficial to obtain governmental approval and commitment for the 
sustainability of DIPoH. Moreover, the approach of establishing a forum involving NTEs to provide their technical opinions to the political stakeholders to make evidence-informed political decisions is in line with the definition of evidence in health established by the European Advisory Committee on Health Research (EACHR) as "findings from research and other knowledge that may serve as a useful basis for decision-making in public health and health care" [12].

Furthermore, the involvement of both, decisionmakers and technical boards, is in line with the WHO Regional Committee for Europe 66th session of September 2016 that declared:

"To improve the linkages between available evidence and policies, producers of knowledge (researchers) and users of knowledge (policy and decision-makers) need to have opportunities and a formal forum for exchange" [13]. Although in InfAct the TDs and the AoM meetings were arranged in different meetings, both boards had the opportunity to interact: indirectly through documentation that included summary meetings (Fig. 1) (InfAct Deliverables (https://www.inf-act.eu/assemblymembers)) [9]; and by the direct exchange of information and recommendations at national level, especially through the set up of National Nodes by InfAct [14]. At TDs level, the analyses of the technical aspects of InfAct outcomes was essential to agree on the fact that the proposal was beneficial and necessary for each country. With this information, the political decision-makers participating in the AoM could focus on the necessary resources and the expected national returns to decide on its political commitment.

Third, the multi-sectorial approach included the involvement of political representatives both from the $\mathrm{MoH}$ and MoR; because a possible obstacle for the development of DIPoH was that in many countries, the research responsibility and funding is within the MoR while a Health related Research Infrastructure falls in the responsibility of $\mathrm{MoH}$. By bringing together those representatives from the same country to the AoM, they were able to discuss their different perspectives and come together with a consensual decision about DIPoH. In the AoM meetings, it was also emphasized that highlevel research (interest of MoR) would also support the needs and interests of $\mathrm{MoH}$.

Fourth, with the involvement of technical and political national representatives, written commitment for the future sustainability of the infrastructure was obtained. At the present time, eleven countries provided letters of political support, three countries provided expression of financial commitment and sixteen institutions supported the project signing the Memorandum of Understanding, for DIPoH to be added to the ESFRI 2021 roadmap.

\section{Limitations}

The implementation of the TDs and AoMs in InfAct has shown several limitations. First, the involvement and participation of high political representatives from 28 EU-MSs and 5 EEA countries was complex due to their agendas. However, participation of the $75 \%$ of the countries was achieved. Second, the country representative was not always the same person for all the AoM meetings. Third, due to COVID-19 travel restrictions, three out of five AoMs and one out of two TDs were held virtually. Finally, despite the positive assessment by TDs and the AoM representatives, most of InfAct's outcomes were not always translated to a MS's political commitment for DIPoH's ESFRI roadmap application.

\section{Conclusion}

Both boards described here, the TDs and the AoM, provided different but complementary and necessary inputs. The TDs focused mainly on added value for HI, feasibility aspects, and new technical and scientific adaptation required from current systems. At the same time, the AoM was more oriented towards resources, necessary country commitments, and national benefits. The outcomes of both boards provided the necessary advice and political support needed for DIPoH, and they are advisable for any EU population health purpose requiring MSs engagement.

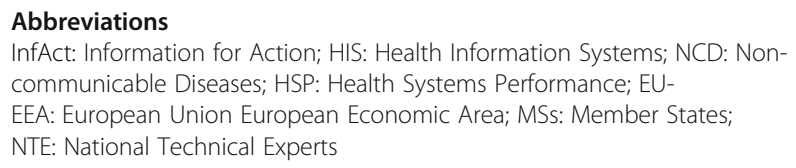

\section{Acknowledgements}

We gratefully acknowledge the contribution of all beneficiaries and stakeholders from the Joint Action Project 801553 Information for Action InfAct.

Likewise, we acknowledge the contributions of the country representatives of the Assembly of Members and the Technical Dialogues from the EU/EEA MSs that participated in the InfAct Project.

\section{Authors' contributions \\ INZ, APM, and RSS and formulated the research question and conceived the study design. INZ, APM, RSS, PB, LA, HVO, HT, MT, LP, RH, AG, LL, TZ, SS, and $\mathrm{JV}$ acquired the data. INZ, APM, and RSS analyzed the results. INZ, APM, and RSS and were major contributors in writing the manuscript. INZ, PB, LA, HVO, $H T, M T, L P, R H, A G, L L, T Z, S S$, and JV critically reviewed the manuscript. All authors read and approved the final manuscript.}

\section{Funding}

European Union's Health Programme (2014-2020) Project 801553 InfAct. The funders had no role in the design, collection, analysis, interpretations, or writing of this work.

\section{Availability of data and materials}

All data generated or analyzed during this study are available from InfAct and in the following links:

www.inf-act.eu

https://www.inf-act.eu/assembly-members 


\section{Declarations}

\section{Ethics approval and consent to participate}

After consultation with the Research Ethics Committee of the Institute of Health Carlos III it was decided that the study needed no formal approval because data collection did not involve human participants or human data.

\section{Consent for publication}

Not applicable.

\section{Competing interests}

HVO is co-authors of this paper and the editor in chief of "Archives of Public Health." RH is co-author of this paper and the section editor of "health information system" of "Archives of Public Health."

The rest of the authors declare that they have no competing interests.

\section{Author details}

${ }^{1}$ National School of Public Health, Instituto de Salud Carlos III. Av/ Monforte de Lemos 5, 28029 Madrid, Spain. ${ }^{2}$ Department of Epidemiology and Public Health, Scientific Institute of Public Health. Sciensano, Brussels, Belgium. ${ }^{3}$ Department of Public Health and Primary Care, Faculty of Medicine, Ghent University, Ghent, Belgium. ${ }^{4}$ Department of Public Health and Welfare, Finnish Institute for Health and Welfare (THL), Mannerheimintie 166, 00271 Helsinki, Finland. ${ }^{5}$ National Institute for Public Health and the Environment (RIVM), Bilthoven, Netherlands. 'Department of Cardiovascular,

Endocrine-metabolic Diseases and Aging, Istituto Superiore di Sanità (ISS), Via Giano della Bella, 34, 00161 Rome, Italy. ${ }^{7}$ Santé Publique France,

Saint-Maurice, France. ${ }^{8}$ Institute of Hygiene and Tropical Medicine, Universidade NOVA de Lisboa, Lisbon, Portugal. ${ }^{9}$ Instituto de Medicina Preventiva e Saúde Pública, Faculdade de Medicina da Universidade de Lisboa, 1649-028 Lisbon, Portugal. ${ }^{10}$ Robert Koch Institute, Berlin, Germany.

${ }^{11}$ Croatian Institute of Public Health (CIPH), Zagreb, Croatia.

Received: 21 June 2021 Accepted: 7 January 2022

Published online: 17 January 2022

\section{References}

1. Council of the European Union. Council conclusions on the "Reflection process on modern, responsive and sustainable health systems" Employment, social policy, health and consumer affairs Council meeting. 2013. https://www.consilium.europa.eu/uedocs/cms_data/docs/pressdata/ en/lsa/140004.pdf Accessed 22 April 2021

2. The BRIDGE Health project Bridging Information and Data Generation for Evidence-based Health policy and research. Bridge Health: Concept Paper Technical Report BRIDGE Health NWP1_2016_03. 2018. https://www. bridge-health.eu/sites/default/files/Technical\%20Report\%20WP1_2016_03_ Concept\%20Paper_final_V2_0.pdf Accessed 23 May 2021.

3. Bogaert $P$, van Oers H. Van Oyen H; for BRIDGE health. Towards a sustainable EU health information system infrastructure: a consensus driven approach. Health Policy. 2018 Dec;122(12):1340-7. https://doi.org/10.1016/j. healthpol.2018.10.009.

4. Bogaert $P$, Van Oyen $H$, for BRIDGE health. An integrated and sustainable EU health information system: national public health institutes' needs and possible benefits. Arch Public Health. 2017;75:3. https://doi.org/10.1186/s13 690-016-0171-7.

5. Public Health Evaluation and Impact Assessment Consortium (PHEIAC) Evaluation of the use and impact of the European Community Health Indicators ECHI by Member States. Final report. 2013. https://ec.europa.eu/ health/sites/health/files/indicators/docs/echi_report_v20131031.pdf Accessed 22 April 2021.

6. Joint Action on Health Information. Information for Action (InfAct). A Joint Action on Health Information. Information for Action (InfAct). 2020. www. inf-act.eu Accessed 22 April 2021.

7. Joint Action on Health Information. Information for Action (InfAct). A Distributed Infrastructure on Population Health. 2020. https://www.inf-act. eu/sites/inf-act.eu/files/2020-01/Booklet.pdf Accessed 22 April 2021.

8. Joint Action on Health Information. Information for Action (InfAct). Fact Sheets. 2020. https://www.inf-act.eu/sites/inf-act.eu/files/2021-04/InfA ct\%20Deliverable\%204.3\%20Fact\%20Sheets_combined.pdf Accessed 10 June 2021.
9. Joint Action on Health Information. Information for Action (InfAct). Assembly of Members. 2021. https://www.inf-act.eu/assembly-members Accessed 5 May 2021.

10. Population Health Information Research Infrastructure (PHIRI). The PHIR project. 2020. https://www.phiri.eu/ Accessed 22 April 2021.

11. Joint Action on Health Information. Information for Action (InfAct). WP 10 : Assessing and piloting interoperability for public health policy. 2021. https:// www.inf-act.eu/wp10 Accessed 5 May 2021.

12. Banta HD. European advisory committee on Health Research (EACHR) Considerations in defining evidence for public health: the European advisory committee on Health Research World Health Organization regional office for Europe. Int J Technol Assess Health Care. 2003;19(3):559-72. https://doi.org/10.1017/S0266462303000515.

13. WHO Regional Committee for Europe. 66th session. Action plan to strengthen the use of evidence, information and research for policy-making in the WHO European Region. 2016. https://www.euro.who.int/_data/a ssets/pdf_file/0006/314727/66wd12e_EIPActionPlan_160528.pdf. Accessed 22 April 2021

14. Joint Action on Health Information. Information for Action (InfAct). National Nodes meeting: from InfAct to PHIRI. 2021. https://www.inf-act.eu/events/na tional-nodes-meeting-infact-phiri Accessed 5 May 2021.

\section{Publisher's Note}

Springer Nature remains neutral with regard to jurisdictional claims in published maps and institutional affiliations.
Ready to submit your research? Choose BMC and benefit from:

- fast, convenient online submission

- thorough peer review by experienced researchers in your field

- rapid publication on acceptance

- support for research data, including large and complex data types

- gold Open Access which fosters wider collaboration and increased citations

- maximum visibility for your research: over $100 \mathrm{M}$ website views per year

At BMC, research is always in progress.

Learn more biomedcentral.com/submissions 